\title{
BMJ Open Acupuncture and PC6 stimulation for the prevention of postoperative nausea and vomiting in patients undergoing elective laparoscopic resection of colorectal cancer: a study protocol for a three-arm randomised pilot trial
}

\author{
Kun Hyung Kim, ${ }^{1}$ Dae Hun Kim, ${ }^{2}$ Ji Min Bae, ${ }^{2}$ Gyung Mo Son, ${ }^{3}$ Kyung Hee Kim, ${ }^{3}$ \\ Seung Pyo Hong, ${ }^{2}$ Gi Young Yang, ${ }^{1}$ Hee Young Kim ${ }^{4}$
}

To cite: Kim KH, Kim DH, Bae JM, et al. Acupuncture and PC6 stimulation for the prevention of postoperative nausea and vomiting in patients undergoing elective laparoscopic resection of colorectal cancer: a study protocol for a three-arm randomised pilot trial. BMJ Open 2017;7:e013457. doi:10.1136/bmjopen-2016013457

- Prepublication history and additional material is available. To view please visit the journal (http://dx.doi.org/ 10.1136/bmjopen-2016013457).

Received 13 July 2016 Revised 18 November 2016 Accepted 15 December 2016

CrossMark

For numbered affiliations see end of article.

Correspondence to Professor Hee Young Kim; yuvi1981@naver.com

\section{ABSTRACT}

Introduction: This study aims to assess the feasibility of acupuncture and a Pericardium 6 (PC6) wristband as an add-on intervention of antiemetic medication for the prevention of postoperative nausea and vomiting (PONV) in patients undergoing elective laparoscopic colorectal cancer resection.

Methods and analysis: A total of 60 participants who are scheduled to undergo elective laparoscopic resection of colorectal cancer will be recruited. An enhanced recovery after surgery protocol using standardised antiemetic medication will be provided for all participants. Participants will be equally randomised into acupuncture plus PC6 wristband (Acupuncture), PC6 wristband alone (Wristband), or no acupuncture or wristband (Control) groups using computergenerated random numbers concealed in opaque, sealed, sequentially numbered envelopes. For the acupuncture combined with PC6 wristband group, the embedded auricular acupuncture technique for preoperative anxiolysis and up to three sessions of acupuncture treatments with manual and electrical stimulation within 48 hours after surgery will be provided by qualified Korean medicine doctors. The PC6 wristband will be applied in the Acupuncture and Wristband groups, beginning 1 hour before surgery and lasting 48 hours postoperatively. The primary outcome will be the number of participants who experience moderate or severe nausea, defined as nausea at least 4 out of 10 on a severity numeric rating scale or vomiting at 24 hours after surgery. Secondary outcomes, including symptom severity, participant global assessments and satisfaction, quality of life, physiological recovery, use of medication and length of hospital stay, will be assessed. Adverse events and postoperative complications will be measured for 1 month after surgery.

Ethics and dissemination: All participants will provide written informed consent. The study has been approved by the institutional review board (IRB). This pilot trial will inform a full-scale randomised trial of

\section{Strengths and limitations of this study}

- The study is the first randomised pilot trial in South Korea testing the feasibility of acupuncture combined with a Pericardium 6 (PC6) wristband in the context of the perioperative care of patients undergoing elective laparoscopic colorectal cancer resection.

- The study is focused on the role of adjuvant acupuncture combined with PC6 stimulation, which few previous studies addressed.

- Methodological rigour, including adequate concealment of random allocation, and prospective trial registration aim to the reduce risk of bias.

- The study aims to guide a future full-scale pragmatic randomised trial to assess the overall effectiveness of acupuncture combined with PC6 for the prevention of postoperative nausea and vomiting.

- Unblinded participants and treatment providers as well as the possibility of unblinding of outcome assessors are likely to result in high risk of performance and detection bias.

acupuncture combined with PC6 stimulation for the prevention of PONV in patients undergoing elective laparoscopic colorectal cancer surgery.

Trial registration number: NCT02509143.

\section{INTRODUCTION}

Postoperative nausea and vomiting (PONV) are significantly distressing to patients and may delay recovery after surgery. The incidence of PONV varies from $\sim 30-50 \%$ of patients undergoing surgery, whereas it reaches up to $80 \%$ of high-risk patients. ${ }^{1}$ Multimodal approaches to managing PONV 
include pharmacological and non-pharmacological interventions, including Pericardium 6 (PC6) acupuncture point stimulation, which has been suggested by consensus expert guidelines. ${ }^{1}$ A recently updated Cochrane Review including a total of 4858 participants in 40 randomised trials found a low quality of evidence of a consistent benefit of PC6 stimulation over placebo for preventing PONV with minimal adverse events; the risk of PONV was similar between PC6 stimulation and antiemetic drugs. ${ }^{2}$ In another systematic review including a total of 2534 participants in 30 trials, there was low-quality evidence for the effectiveness of PC6 combined with other acupuncture points compared with PC6 alone or no acupuncture. ${ }^{3}$ Several preclinical experimental studies showed the potential effects of acupuncture on gastric myoelectrical activity, vagal modulation and cerebellar vestibular activities in brain imaging studies, all of which may be associated with prevention of PONV. ${ }^{4}$ What remains incompletely understood and requires further investigation includes: (1) the effects of PC6 within multimodal prophylaxis of PONV (ie, additive or synergistic effects of PC6 acupuncture on antiemetic use) $;^{2}$ and (2) the combined effects of PC6 and other acupuncture points compared with PC6 alone (ie, dose-response relationship of acupuncture stimulation). ${ }^{2}{ }^{3}$ In our previous systematic review of the effects of acupuncture for recovery after colorectal cancer surgery, there were no significant overall differences in PONV between the acupuncture and control groups (placebo or no acupuncture). ${ }^{5}$ However, a small sample size and significant heterogeneity prohibit drawing conclusions without further evidence from high-quality studies. This study specifically aims to address the gaps in current evidence by developing the research questions as follows:

1. Is it feasible to conduct a parallel group, equally allocated superiority randomised controlled trial of acupuncture to prevent PONV in a standardised multimodal perioperative care protocol in patients with colorectal cancer?

2. Does the combined use of PC6 and other acupuncture points show more benefit in the management of PONV than PC6 stimulation alone?

3. Does acupuncture (as PC6 stimulation or PC6 combined with other acupuncture points) combined with antiemetic drugs provide more benefit in the management of PONV than antiemetic drugs alone?

\section{METHODS AND ANALYSIS}

\section{Study design}

\section{Randomisation and allocation concealment}

An independent biostatistician generated a block random sequence using the Excel spreadsheet program. An independent hospital staff member will prepare sequentially numbered, opaque, sealed envelopes to ensure concealment of the allocation sequence. A research assistant will open the envelope in front of the patient, who will provide written informed consent.

\section{Study context}

This study will be a pilot parallel three-group randomised controlled trial performed in the inpatient ward of Pusan National University Yangsan Hospital, Yangsan, South Korea. This tertiary hospital covers urban and rural areas of Yangsan, which had a population of $\sim 300000$ people in 2015. ${ }^{6}$ The annual surgical volume for elective laparoscopic resection of colorectal cancer was 353 in the most recent 12 months. A total of nine investigators will be involved, including a specialist colorectal surgeon (KMS) with 12 years of clinical experience, an anaesthesiologist dedicated to the perioperative anaesthesia care for study participants (HYK), two Korean medicine doctors (KMDs) for acupuncture treatments (KHK and GYY), three research assistants (DHK, JMB and SPH), a physician assistant (KHK) and a study coordinator for participant screening and data collection. Trained nurses and dieticians in the inpatient ward had practised an enhanced recovery after surgery (ERAS) programme for patients undergoing colorectal cancer resection. ${ }^{7}$ Acupuncture will be provided in the inpatient room.

\section{Selection of target conditions}

Patients undergoing elective laparoscopic surgery for colorectal cancer resection will be our main target population. We did not consider open resection or other types of abdominal surgery due to possible confounding from variations in perioperative care protocols (eg, variations in the use of opioid analgesics or in postoperative recovery care). The Cochrane Review of acupuncture for PONV does not include trials conducted in patients with colorectal cancer, ${ }^{2}$ although the type of surgery is not an established risk factor for PONV. ${ }^{1}$ We assumed that relatively homogenous participants will be needed to reduce potential unknown sources of heterogeneity that may affect study results.

\section{Pretreatment assessment and the participant recruitment process}

Patient recruitment began on 1 September 2015. Patients undergoing elective laparoscopic resection of colorectal cancer were screened at the time of outpatient consultation by the study surgeon and the physician assistant nurse. If the patient is deemed eligible and shows interest in study participation, the research assistant will contact the patient to provide full information regarding study participation and to obtain written informed consent. Those patients who agree to participate and meet the full eligibility criteria will be allocated to one of three groups, namely: (1) PC6 and other acupuncture group (Acupuncture group); (2) PC6 stimulation group (Wristband group); and (3) no acupuncture group (Control group), in a 1:1:1 ratio. The schedule of 
trial enrolment, interventions and assessment are presented in table 1. Figure 1 illustrates the trial flow chart.

\section{Eligibility criteria}

\section{Eligible}

1. patients aged $18-80$ years;

2. patients undergoing elective laparoscopic surgery for colorectal cancer resection (right hemicolectomy, left hemicolectomy, anterior resection with primary anastomosis, or low anterior resection with loop ileostomy for faecal diversion);

3. American Society of Anaesthesiologists Grade 1 to 2.

\section{Ineligible}

1. comorbidities that may affect surgical outcome (eg, chronic kidney disease, chronic liver disease, cardiopulmonary failure, diabetes with complications or inflammatory bowel disease);

2. use of emetogenic/antiemetic medication within 24 hours before surgery;

3. history of emetic episodes after administration of antibiotics;

4. expected use of mechanical ventilation;

5. cognitive impairment that could affect the patient's ability to complete the outcome assessments;

6. previous history of stroke or epilepsy;

7. pregnancy;

8. previous history of sensitivity reaction to acupuncture;

9. unable to cooperate with acupuncture treatments;

10. pacemaker implantation;

11. patients who had received Korean medical treatments (acupuncture, moxibustion, cupping or herbal medicine) within 2 weeks;

12. patients who had participated in other trials within 3 months.

We will exclude patients if unexpected conversion to open surgery occurs.

\section{Blinding of participants, practitioners, other relevant} healthcare staff, outcome assessors and data analysers

This is an open-label study comparing acupuncture plus a PC6 wristband combined with the ERAS programme (Acupuncture group) versus a PC6 wristband combined with the ERAS programme (Wristband group) versus the ERAS programme alone (Control group). The participants, acupuncture practitioners, a study surgeon, a study anaesthetist and other hospital staff will be aware of group allocation. Two trained outcome assessors will remain blinded to group allocation and collect physiological and subjective outcome data before or after discharge, although a possibility of unblinding cannot not be excluded if the patient's wristband is noticed by the assessors within postoperative 48 hours. An independent, blinded biostatistician will perform the statistical analyses.

\section{Components of the ERAS protocol}

All study participants will receive perioperative care based on a standardised ERAS protocol. Components of our ERAS protocol met 14 of 17 key elements in a systematic review $^{8}$ (table 2). For acupuncture and the PC6 wristband group (acupuncture group and wristband group), acupuncture and/or a PC6 wristband will be applied as additional components of ERAS. Daily guidelines for perioperative care in the ERAS protocol are presented in online supplementary appendix 1 .

\section{Anaesthetic process}

A standardised anaesthetic protocol will be applied to all patients. General anaesthesia will be induced with intravenous administration of thiopental $4-5 \mathrm{mg} / \mathrm{kg}$, rocuronium $\quad 0.6-0.9 \mathrm{mg} / \mathrm{kg}$ and remifentanil $0.5-1 \mathrm{mg} / \mathrm{kg}$. Adequate hypnosis, analgesia and muscle relaxation will be maintained by sevoflurane (2-3 vol $\%$ ), a titrated dose of remifentanil, and rocuronium under monitoring of the bispectral index. Standard patient monitoring including ECG, pulse oximetry, capnography, noninvasive blood pressure and respiratory sevoflurane concentrations will be performed. The study anaesthesiologist will infuse multiple electrolyte solutions (Plasma-Lyte 148 ( $\mathrm{pH}$ 7.4)) intravenously and control the infusion dose of the fluids based on calculated preoperative deficits, third space losses, urine output and estimated intraoperative blood loss. After $\mathrm{CO}_{2}$ insufflation, the patients will be placed in the Trendelenburg position and tilted right or left. Mechanical ventilation will be performed, and end-tidal $\mathrm{CO}_{2}$ will be maintained between 35 and $40 \mathrm{~mm} \mathrm{Hg}$ by adjusting respiratory rate, tidal volume and peak pressure. A loading dose of $0.1 \mathrm{~mL} / \mathrm{kg}$ and intravenous injection of ramosetron $0.3 \mathrm{mg}$ will be administered at the end of surgery. Pyridostigmine $10 \mathrm{mg}$ and glycopyrrolate $0.4 \mathrm{mg}$ will be administered intravenously to reverse the residual effect of the muscle relaxant. The patients will be transferred to the postanaesthesia care unit (PACU) after tracheal extubation. In the PACU, intravenous fluid therapy (Plasma-Lyte 148 ( $\mathrm{pH}$ 7.4) solution) will be continued at a maintenance dose. Intravenous patient-controlled analgesia (IV-PCA) will be connected in the PACU.

\section{Study interventions}

\section{Timing of acupuncture stimulation}

Variation in the timing of acupuncture was suggested as a source of heterogeneity in previous meta-analyses of acupuncture for PONV. ${ }^{2}{ }^{3}$ No clear evidence exists for the optimal timing of acupuncture (ie, preoperative, intra operative or postoperative).$^{2}$ Few trials have directly compared different timings of acupuncture for better prevention of PONV. Our systematic review found that trials in which acupuncture was administered postoperatively showed inconsistent results in the prevention of PONV in patients with colorectal cancer. ${ }^{5}$ Although it is plausible that sensory stimulation triggering favourable physiological responses to prevent PONV would not be 
Table 1 Measurements of patient characteristics and outcomes Enrolment Allocation Pre-OP Intra-OP PACU 6 hours 12 hours 24 hours 48 hours Discharge 2 weeks 4 weeks

Enrolment

Informed consent

Assessment of eligibility

Randomisation

Interventions

Embedded acupuncture

Body acupuncture

PC6 wristband stimulation

Standard antiemetics

Assessments

Preoperative anxiety

Pain intensity NRS

Postoperative nausea and

vomiting

Physiological recovery ${ }^{\star}$

Quality of life

Patient satisfaction for

management of PONV

Patient's global assessment of

recovery after surgery

Use of medication

Harms/adverse events

Postoperative complications

Length of hospital stay

\section{$\mathrm{O}$}

O

O

$\mathrm{O}$

O

O

O

O

O

$\mathrm{O}$

O

$\begin{array}{ll}0 & 0 \\ 0 & 0 \\ 0 & 0\end{array}$

0

$\begin{array}{ll}0 & 0 \\ 0 & 0\end{array}$

O

O

O

$\begin{array}{llllll}0 & 0 & 0 & 0 & 0 & 0 \\ 0 & 0 & 0 & 0 & 0 & 0 \\ 0 & 0 & 0 & 0 & 0 & 0 \\ & & & & & 0\end{array}$

${ }^{*}$ Assessment of physiological recovery includes measuring time to first flatus, time to tolerate soft diet, time to first defaecation, time to independent walking, time to first removal of Foley catheter, the number of reinsertions of Foley catheter, the number of clean intermittent catheterisations and the number of insertions of nasogastric tube.

NRS, numerical rating scale; OP, operation; PACU, postanaesthesia care unit; PONV, postoperative nausea and vomiting. 


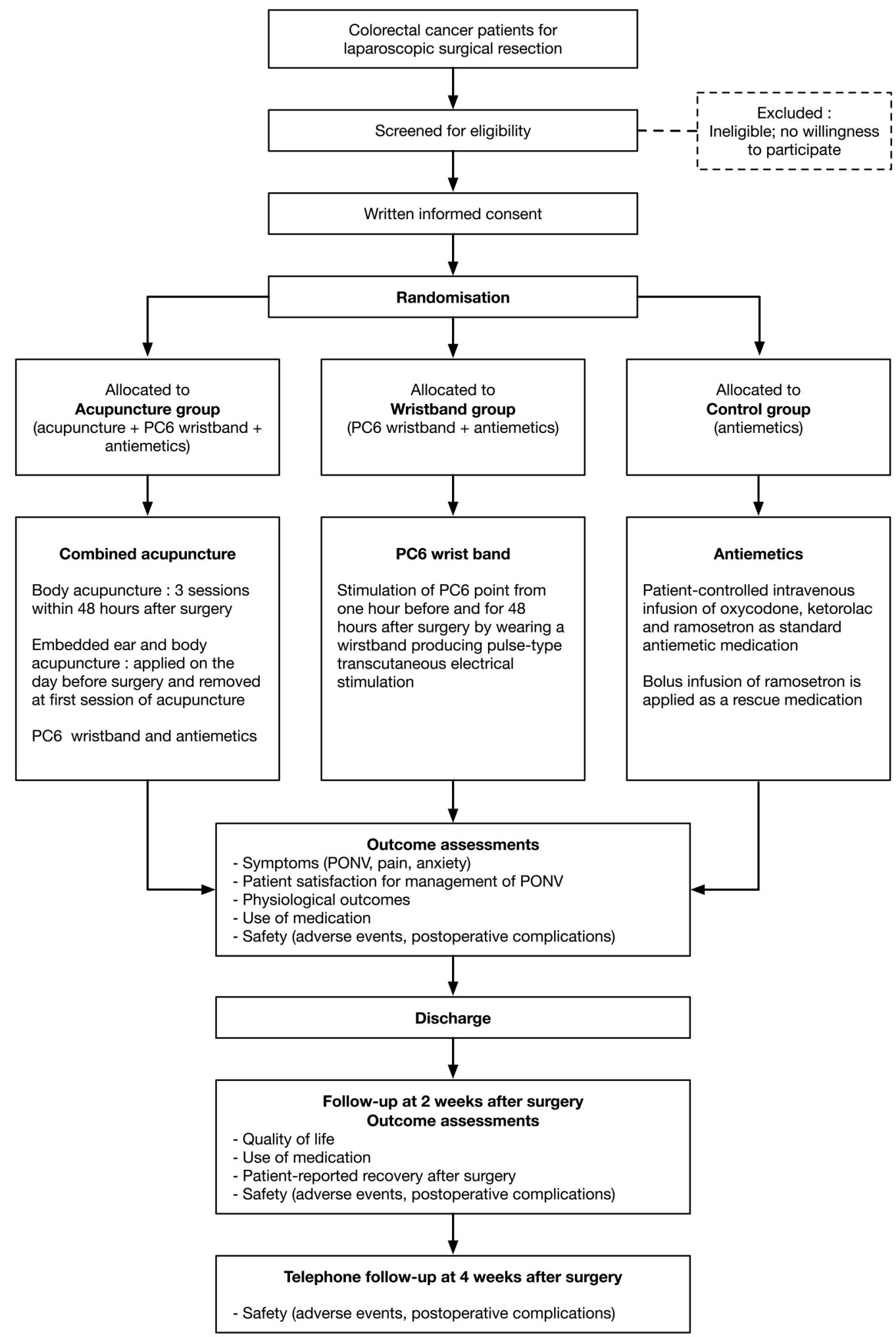

Figure 1 Trial flow chart.

obtained under general anaesthesia, ${ }^{9}{ }^{10}$ favourable effects of the perioperative administration of acupuncture, including intraoperative stimulation, were observed in some trials. ${ }^{11}{ }^{12}$ While specific recommendations cannot be made due to a paucity of evidence, the perioperative administration (ie, preoperative, intraoperative and postoperative) of acupuncture stimulation was chosen for the maximum possible benefit in our trial.

\section{Perioperative embedded acupuncture}

An embedded acupuncture technique for preoperative anxiolysis suggested in a recent systematic review ${ }^{13}$ will 
Table 2 Components of an enhanced recovery program

Components

Enhanced recovery

included

For all three groups*

1 Preoperative education and stoma training

2 Carbohydrate loading preoperatively and avoidance of prolonged starving

3 Use of preoperative probiotics

$4 \quad$ No mechanical bowel preparation

5 No premedication $\dagger$

6 Goal-directed perioperative fluid administration

7 High perioperative $\mathrm{O}_{2}$ concentrations $\mathrm{O}$

8 Maintenance of normothermia

9 Epidural analgesia

10 Laparoscopic surgery or transverse $\mathrm{O}$ incisions

11 Avoidance of nasogastric tubes $\mathrm{O}$

12 Avoidance of drains

13 Avoidance of opioid analgesia

14 Use of postoperative laxatives

15 Early removal of bladder catheter

16 Enforced early mobilisation

17 Enforced early postoperative oral feeding

For Acupuncture and/or Wristband group

18 PC6 wristband for prevention of PONV (for both groups)

19 Ear acupuncture for preoperative anxiolysis (for Acupuncture group)

20 Body acupuncture for prevention of PONV/recovery after surgery (for both groups)

*Seventeen components reflect the key elements of enhanced recovery after surgery in colonic surgery, suggested by Wind et al. ${ }^{8}$

†Premedication is provided according to the local anaesthetic protocol (intramuscular injection of glycopyrrolate and midazolam).

PONV, postoperative nausea and vomiting.

be applied to the bilateral acupuncture points of Liver (LI4), Heart 7 (HT7), Stomach (ST36), Yin-Tang, ear Shen-Men and ear sympathetic using the superficial needle (Dongbang Press Needles, 0.18×1.3 mm, Dongbang) the day before surgery and will be removed at the first session of acupuncture on the day of surgery. These treatments will be applied only to the patients in the Acupuncture group.

\section{Perioperative PC6 wristband stimulation}

Stimulation of PC6 points will be maintained from 1 hour before and until 48 hours after surgery by a wristband that produces pulse-type transcutaneous electrical stimulation (Relief Band, Model WB-DL150 Hour Device, Neurowave Medical Technologies LLC, Chicago, Illinois, USA). A study KMD will educate the patients regarding the accurate wear of PC6. The PC6 wristband will be applied to the patients in the Acupuncture group and the Wristband group.

\section{Postoperative body acupuncture}

Three sessions of acupuncture will be provided within 48 hours after surgery because this time window is the most distressing period in terms of PONV. The first session will be performed within 2 hours after surgery. Acupuncture needles $(0.25 \times 30 \mathrm{~mm}, 0.25 \times 40 \mathrm{~mm}$ or $0.30 \times 60 \mathrm{~mm}$, Dongbang) inserted on the body points of Stomach 36 (ST36), Stomach 37 (ST37), Liver 3 (LR3), Large Intestine 11 (LI11), Large Intestine 4 (LI4), Spleen 6 (SP6), Spleen 4 (SP4), Pericardium 6 (PC6), Heart 8 (HT8) and Gall Bladder 41 (GB41) will be manually stimulated to achieve De-qi sensation. All points are located in the peripheral extremities to avoid irritating the surgical site. Subsequent electrical stimulation with an alternating frequency of $2-100 \mathrm{~Hz}$, a biphasic square wave and tolerable intensity by the patient (ES-160, Ito, Tokyo, Japan) will be applied to selected points (PC6 to LI4, ST36 to ST37, and bilateral SP6). Electrical stimulation will be not applied on the arm with the IV to not interfere with the intravenous needles by possible muscle twitching. Needles will be retained for $20 \mathrm{~min}$ in each session unless the patient experienced discomfort from the treatment. These acupuncture treatments will be provided to the patients in the Acupuncture group. Full information about the acupuncture treatments is presented in online supplementary appendix 2 .

\section{Antiemetics}

A standardised protocol to prevent PONV was developed by the study anaesthetist (HYK). A mixture of standard antiemetic medication (5-hydroxytryptophan receptor antagonist, ramosetron hydrochloride $0.3 \mathrm{mg}$ ) and analgesics, including a non-steroidal anti-inflammatory drug (NSAID) (ketorolac tromethamine $120 \mathrm{mg}$ ) and a semisynthesised opioid (oxycodone $20 \mathrm{mg}$ ), will be infused by intravenous patient-controlled analgesia ( $1 \mathrm{~mL}$ bolus $/ 20 \mathrm{~min}$ lockout, $1 \mathrm{~mL} /$ hour continuous infusion). Antiemetics will be provided to all patients.

\section{Rescue medication}

A bolus infusion of ramosetron $0.3 \mathrm{mg}$ will be given as a rescue antiemetic up to two times a day, in the event of nausea $\geq 6 / 10$ on a $0-10$ numeric rating scale (NRS) (nausea severity scale), vomiting or the request of the patient regardless of the severity of nausea. Rescue antiemetics will be available to all patients. For postoperative pain, additional rescue doses of analgesics will be administered at the patient's request.

\section{Concomitant treatments}

There will be no restriction on the use of other concomitant treatments outside the trial for the pragmatic purpose of this study. Instead, information on 
out-of-study healthcare resources sought by the patient will be collected and descriptively analysed.

\section{Criteria for discontinuing or modifying interventions}

Acupuncture or PC6 stimulation will be modified or discontinued at the patient's request or by the study physician (surgeon, anaesthetist or KMD) to prevent harm or patient discomfort during the recovery process. The antiemetic regimen protocol will be modified or discontinued at the discretion of the study surgeon or anaesthetist if there is a clinically justifiable reason to do so.

\section{Strategies to improve adherence to intervention protocols}

For the Acupuncture and/or PC6 Wristband groups, the study KMD or assistant KMD will visit the patient each day within 48 hours after surgery and ask the patient to report any discomfort during the intervention. The study surgeon, physician assistant (KHK) and anaesthetist will monitor the postoperative recovery process and, where appropriate, modify the intervention or postoperative care to improve the participants' trial adherence.

\section{Rationale of the comparison and the lack of a placebo/ sham intervention group}

According to the recent Cochrane Review, ${ }^{2}$ further study comparing PC6 with placebo will be unlikely to change the established efficacy of PC6 stimulation. Thus, the sham acupuncture will be avoided to prevent duplication of previous evidence. Whether PC6 stimulation combined with antiemetic drugs can show additional benefits compared with antiemetic drugs alone will be suggested as a topic for future research. There is insufficient evidence for higher doses of acupuncture (PC6 and stimulation of additional acupuncture points) compared with PC6 alone, which warrants further research. ${ }^{3}$ Therefore, higher doses of acupuncture (ear, body acupuncture and PC6 wristband stimulation) will be compared with PC6 wristband stimulation.

\section{Outcomes}

\section{Primary outcomes}

The number of patients who experience moderate or severe nausea (ie, at least 4 points of nausea on $0-10$ NRS) or vomiting during the first 24 postoperative hours will be recorded $^{14}$ (table 2).

\section{Secondary outcomes}

\section{Time window}

Time windows will be divided into five phases: from discharge from the recovery room (hereafter 'after surgery') to 6 hours, from 6 to 12 hours, from 12 to 24 hours, from 24 to 48 hours and from 48 hours until discharge after surgery.

\section{Nausea and vomiting}

The number of participants who experience at least one episode of vomiting as well as the total number of vomiting events will be counted within each time window and at discharge. The number of participants who experience any degree of nausea will be measured at the same time points. Nausea severity will be measured on a NRS of 0 (no nausea at all) to 10 (the worst nausea imaginable) at the same time points.

\section{Pain}

Pain scores on a NRS of 0 (no pain at all) to 10 (the worst pain imaginable) will be assessed at $6,12,24$, and 48 hours after surgery and at discharge.

\section{Patient global assessment and satisfaction}

Patient's global assessment of recovery after surgery will be asked at 2 weeks after surgery. Response options include very much improved, somewhat improved, no change, somewhat worsened and very much worsened. These will serve as patient-reported outcomes after surgery, which reflect the patient's experience of recovery. Patient-reported satisfaction with the management of PONV will be measured at 48 hours after surgery on a 0 to 10 NRS (0: very unsatisfactory, 10 : very satisfactory). Higher patient satisfaction with service quality is associated with better survival outcomes in colorectal and other various types of cancer. ${ }^{15-17}$

\section{Physiological outcomes}

Outcomes reflecting physiological recovery until discharge will be measured. These include time to first flatus, time to tolerate soft diet, time to first defaecation, time to independent walking, number of insertions of nasogastric tube, time to first removal of Foley catheter, the number of reinsertions of Foley catheter and the number of clean intermittent catheterisations. Participants or their caregivers will be asked to fill out the diary throughout the admission. Electronic medical and nursing records will also be reviewed for the completeness of data by blinded assessors.

\section{Quality of life}

Quality of life will be measured by the validated Korean translation of the European Organisation for Research and Treatment of Cancer Quality of Life Questionnaire (EORTC QLQ) C30 V.3.0 at 2 weeks after surgery (postdischarge outcome). ${ }^{18} 19$ This questionnaire is comprised of five functional scales, three symptom scales, six symptom items and a single global health status item. The scales range in score from 0 to 100 . Higher scores represent better outcomes on functional scales and a global health status item, whereas lower scores refer to better outcomes in symptom scales and items. ${ }^{20}$

\section{Preoperative anxiety}

A physician assistant nurse will measure the participant's preoperative anxiety in the surgical waiting room just before the induction of anaesthesia, using a 0 (no anxiety at all) to 10 (extreme anxiety) NRS. Although preoperative anxiety is not a significant risk factor of 
PONV, it affects a patient's postoperative anxiety, pain, analgesic consumption, length of hospital stay and satisfaction with their postoperative care and treatments. ${ }^{21}$

\section{Use of medication}

The use of medication including, but not limited to, antiemetics or analgesics will be assessed at 2 weeks after surgery and measured based on electronic medical charts. Self-reported use of additional medication or healthcare resources (such as outpatient clinic visits) will be measured at 2 weeks after surgery.

\section{Length of hospital stay}

The length of hospital stay will be counted by reviewing electronic medical charts.

\section{Adverse events, postoperative complications and serious adverse events after surgery}

All expected and unexpected adverse events will be measured during the allocated intervention process and during the entire study period. The types and frequencies of adverse events will be measured using structured items and an open-ended question. Information on postoperative complications including, but not limited to, wound infection, urinary tract infection, urinary retention, chest infection, other infection and paralytic ileus will be collected within 4 weeks after surgery. Any life-threatening events or serious adverse events (SAEs) occurring within 4 weeks after surgery will be investigated. The complete list of SAE is available on the clinicaltrials.gov database.

\section{Feasibility outcomes}

The feasibility of the study will be measured using two outcomes: (1) the number of patients who completed primary outcome measures, and (2) the number of patients who adhered to the allocated intervention until completion of trial.

\section{Sample size calculation}

This will be a pilot trial to inform the design of a future full-scale randomised trial and will aim to test the feasibility of conducting the study. Recruitment of 20 participants in each group or a total of 60 participants will be deemed sufficient for a pilot trial and will be affordable in our research setting, given available resources and a possible dropout rate of $20 \%$. No prospective sample size calculation was performed.

\section{Data management}

Double data entry and range checks for data values will be performed by trained research assistant after completion of final data collection. A formal data monitoring committee is not needed because the study acupuncture has known minimal risks. ${ }^{22}$ Periodic data monitoring and audit for core aspects of trial conduct such as participant recruitment, outcome collection and reporting of SAEs will be conducted by two independent, trained investigators. The final, anonymised data set will be available to the first author and independent statistician.

\section{Statistical data analysis}

Intention-to-treat population, defined as participants who are initially allocated in the study entry stage, will be the source of primary analyses. A per-protocol population, defined as participants who complete the final post-treatment primary outcome assessment, will serve as the source of sensitivity analyses. Missing data will not be imputed. Relative risk for dichotomous or dichotomised categorical outcomes and mean difference with 95\% CI will be calculated. $\chi^{2}$ or Fisher's exact test will be used for statistical significance testing where appropriate, based on the normality of distribution. Normality of data will be tested by Shapiro-Wilk analysis. Comparisons of descriptive continuous data among groups will be made using the analysis of variance (ANOVA) or Kruskal-Wallis test. For the continuous outcomes, analysis of covariance (ANCOVA) will be performed with the changes in each outcome as a dependent variable, baseline values as a covariate and group as a fixed factor. To assess the trend of changes in outcomes within each group, repeatedmeasures ANOVA will be performed with factors of groups (Acupuncture, Wristband and Control group) and time as separate two-level factors. The Bonferroni correction for multiple comparisons will be made to adjust the $\mathrm{p}$ value. The two-sided overall $\mathrm{p}$ value $<0.05$ will be the level of statistical significance with $90 \%$ power. STATA/SE V.14.1 (StataCorp, College Station, Texas, USA) will be used for the statistical analyses.

\section{Dissemination}

The results of this trial will be presented at scientific workshops and meetings and will be published in peerreviewed journals. For transparency of trial results, the anonymised raw data set will be available based on request to the research team after the publication of study results. All publications and study results will be disseminated to the study participants and personnel.

\section{Written informed consent and study approval}

Each participant will be asked to provide written informed consent. Any modification to the protocol will be reported and approved by the local IRB, and will be communicated with the trial registry, investigators and data monitoring researchers. All participants will receive a financial reimbursement of 30 thousand Korean won ( $\sim$ US\$30) for their time and participation in the trial at the completion of the study.

\section{DISCUSSION}

This study will be the first randomised controlled trial on the use of acupuncture combined with PC6 stimulation for the prevention of PONV after colorectal cancer resection. There is sound evidence that PC6 stimulation is effective for the prevention of PONV. Whether or to what extent the combined use of other points with PC6 could be more beneficial, as well as whether 
acupuncture combined with antiemetics are more beneficial than anti-emetics alone, are suggested as future research areas. Our trial focuses on these gaps of evidence and will inform the design of a full-scale randomised trial to assess the benefits and harms of acupuncture combined with PC6 stimulation and antiemetic drugs. Limitations of this trial will include nonblinding of the study participants and treatment providers, a possibility of unblinding of outcome assessors by occasionally noticing the wristband within postoperative 48 hours and the limited generalisability because of its single-centre nature.

\section{Trial status}

The trial is currently in the recruitment phase. The first patient was randomised on 1 January 2016. Currently (as of 14 November 2016), 58 patients have been completely enrolled in the trial. The expected date of trial completion is 31 December 2016.

\section{Author affiliations}

${ }^{1}$ School of Korean Medicine, Pusan National University, Yangsan, South Korea ${ }^{2}$ Department of Acupuncture \& Moxibustion, Korean Medicine Hospital, Pusan National University, Yangsan, South Korea

${ }^{3}$ Department of Surgery, Pusan National University Yangsan Hospital, Yangsan, South Korea

${ }^{4}$ Department of Anesthesia and Pain Medicine, Pusan National University Yangsan Hospital, Yangsan, South Korea

Contributors KHK and HYK conceived the study. KHK, DHK, GMS, KyHK, GYY and HYK initiated the study design and drafted the protocol. All authors contributed to the refinement of the study protocol and approved the final manuscript. KHK is the guarantor of this manuscript.

Funding This study is supported by a Biomedical Research Institute Grant (2015-00), Pusan National University Hospital (contact information: mri@pnuh.co.kr; 82-51-240-7526).

Disclaimer The funding body has no role in the study design and will not play any role during its execution, analyses, interpretation of the data, or decision to submit the report for publication.

Competing interests None declared.

Patient consent Obtained.

Ethics approval Institutional Review Board (IRB) of Pusan National University Yangsan Hospital (IRB approval number 03-2013-012).

Provenance and peer review Not commissioned; externally peer reviewed.

Data sharing statement Requested data for public purpose or research transparency will be provided (please contact the first or corresponding author).

Open Access This is an Open Access article distributed in accordance with the Creative Commons Attribution Non Commercial (CC BY-NC 4.0) license, which permits others to distribute, remix, adapt, build upon this work noncommercially, and license their derivative works on different terms, provided the original work is properly cited and the use is non-commercial. See: http:// creativecommons.org/licenses/by-nc/4.0/

\section{REFERENCES}

1. Gan TJ, Diemunsch P, Habib AS, et al. Consensus guidelines for the management of postoperative nausea and vomiting. Anesth Analg 2014;118:85-113.

2. Lee A, Chan SK, Fan LT. Stimulation of the wrist acupuncture point PC6 for preventing postoperative nausea and vomiting. Cochrane Database Syst Rev 2015;(11):CD003281.

3. Cheong KB, Zhang JP, Huang $\mathrm{Y}$, et al. The effectiveness of acupuncture in prevention and treatment of postoperative nausea and vomiting - a systematic review and meta-analysis. PLOS ONE 2013;8:e82474.

4. Streitberger K, Ezzo J, Schneider A. Acupuncture for nausea and vomiting: an update of clinical and experimental studies. Auton Neurosci 2006;129:107-17.

5. Kim KH, Kim DH, Kim HY, et al. Acupuncture for recovery after surgery in patients undergoing colorectal cancer resection: a systematic review and meta-analysis. Acupunct Med 2016;34:248-56.

6. http://stat.yangsan.go.kr (accessed 1 Feb 2016).

7. Son KM, Kim KH, Cha SR, et al. Clinical effect of clinical pathway for patients with laparoscopic colon surgery. Journal of Pusan National Univerisity Hospital 2012;31:1-11.

8. Wind J, Polle SW, Fung Kon Jin PH, et al. Systematic review of enhanced recovery programmes in colonic surgery. Br J Surg 2006:93:800-9.

9. Usichenko TI, Hesse T. Appropriate timing and intensity of PC6 stimulation for the prevention of postoperative nausea and vomiting. Acupunct Med 2016;34:70-1.

10. Alraek T, Birch S. Acupuncture effects under anaesthesia and questions about placebo. Acupunct Med 2016;34:

$155-6$.

11. Usichenko TI, Streitberger K. Perioperative acupuncture: why are we not using it? Acupunct Med 2014;32:212-14.

12. Yang $X Y$, Xiao J, Chen $\mathrm{YH}$, et al. Dexamethasone alone vs in combination with transcutaneous electrical acupoint stimulation or tropisetron for prevention of postoperative nausea and vomiting in gynaecological patients undergoing laparoscopic surgery. Br J Anaesth 2015;115:883-9.

13. Bae $\mathrm{H}, \mathrm{Bae} \mathrm{H}, \mathrm{Min} \mathrm{BI}$, et al. Efficacy of acupuncture in reducing preoperative anxiety: a meta-analysis. Evid Based Complement Alternat Med 2014;2014:850367.

14. Apfel CC, Korttila K, Abdalla M, et al. A factorial trial of six interventions for the prevention of postoperative nausea and vomiting. N Engl J Med 2004;350:2441-51.

15. Gupta D, Lis CG, Rodeghier M. Can patient experience with service quality predict survival in colorectal cancer? J Healthc Qual 2013;35:37-43.

16. Gupta D, Rodeghier M, Lis CG. Patient satisfaction with service quality in an oncology setting: implications for prognosis in non-small cell lung cancer. Int J Qual Health Care 2013;25:696-703.

17. Gupta D, Rodeghier M, Lis CG. Patient satisfaction with service quality as a predictor of survival outcomes in breast cancer. Support Care Cancer 2014;22:129-34.

18. Yun YH, Park YS, Lee ES, et al. Validation of the Korean version of the EORTC QLQ-C30. Qual Life Res 2004;13:863-8.

19. Aaronson NK, Ahmedzai S, Bergman B, et al. The European Organization for Research and Treatment of Cancer QLQ-C30: a quality-of-life instrument for use in international clinical trials in oncology. J Natl Cancer Inst 1993;85:365-76.

20. Fayers PM, Aaronson NK, Bjordal K, et al. The EORTC QLQ-C3O scoring manual. 3rd edn. European Organisation for Research and Treatment of Cancer, 2001.

21. Hansen MV, Halladin NL, Rosenberg J, et al. Melatonin for pre- and postoperative anxiety in adults. Cochrane Database Syst Rev 2015; (4):CD009861.

22. Witt CM, Pach D, Brinkhaus B, et al. Safety of acupuncture: results of a prospective observational study with 229,230 patients and introduction of a medical information and consent form. Forsch Komplementmed 2009;16:91-7. 